\title{
Synthesis and Characterization of Al-Doped Zinc Oxide Films by an Radio Frequency Magnetron Sputtering Method for Transparent Electrode Applications
}

\author{
Jae Keun Seo, Ki-han Ko, Hyung Jun Cho, and Won Seok Choi ${ }^{\dagger}$ \\ Department of Electrical Engineering, Hanbat National University, Daejeon 305-714, Republic of Korea \\ Mungi Park and Kyung-han Seo \\ LG Display Co., Ltd., Paju 413-811, Republic of Korea \\ Young Park \\ Korea Railroad Research Institute, Uiwang 437-757, Republic of Korea \\ Dong-Gun Lim \\ Department of Electronic Engineering, Chungju Natinal University, Chungju 380-702, Republic of Korea \\ Received November 6 2009, Revised January 7 2010, Accepted January 192010
}

\begin{abstract}
In this study, transparent and conductive Al-doped zinc oxide (AZO) films were prepared on a glass substrate by an radio frequency (RF) magnetron sputtering method using a 150-nm-thick AZO target (Al: 2 wt.\%) at room temperature. We investigated the effects of RF power between 100-350 W (in steps of $50 \mathrm{~W}$ ) on the structural, electrical, and optical properties of the AZO films. The thickness and cross-sectional images of the films were observed by field emission scanning electron microscopy. The thicknesses of all films were kept constant at $150 \mathrm{~nm}$ and grown on a glass substrate. The grain sizes of the AZO films were determined with the X-ray diffraction by using the Scherrer' equation, and their electrical properties were investigated using a Hall effect electronic transport measurement system. The transmittance of the AZO films was also measured by an ultraviolet-visible spectrometer.
\end{abstract}

Keywords: Al-doped zinc oxide, Radio frequency magnetron sputtering method, Radio frequency power, Field emission scanning electron microscopy, X-ray diffraction

\section{INTRODUCTION}

Transparent conducting oxide (TCO) films have been widely used for mobile display applications, such as organic lighting diodes, liquid crystal displays (LCD), micro displays, and solar cells [1]. Until now, indium tin oxide (ITO) films have been popular amongst TCO materials because of high stability and low resistivity. However, ITO meets the requirements of transparent conducting thin films which has made it a commodity and is in short supply, as indium is somewhat rare

\footnotetext{
+ Author to whom corresponding should be addressed: electronic mail: wschoi@hanbat.ac.kr
}

and expensive [2].

Recently, zinc oxide ( $\mathrm{ZnO}$ ) has been considered an ideal alternative material due to its lower cost and wide availability [3], [4]. Recent research reveals that Al-, B-, Ga-, and Mndoped $\mathrm{ZnO}$ films show low resistivity and high transmittance [5]-[8]. Also, to obtain high-quality $\mathrm{ZnO}$ films, a variety of techniques may be used, such as RF magnetron sputtering, pulse laser deposition (PLD), sol-gel, and DC sputtering [9][12]. A radio frequency (RF) magnetron sputtering method is generally used for TCO film synthesis. For some product applications, the TCO must be synthesized at lower temperatures, such as under $200^{\circ} \mathrm{C}[13]$.

In this study, we investigated the effects of RF power on the structural, electrical, and optical properties of Al-doped 
ZnO (AZO) films. We used an AZO target (Al: 2 wt.\%) to synthesis AZO films, which were deposited at room temperature. The thickness of all the films was kept constant at 150 $\mathrm{nm}$, because this is the thickness of TCO electrodes used in LCD products.

\section{EXPERIMENTS}

The AZO films were deposited on a glass (OA-10G; Nippon Electronic Glass Co., Ltd., Tokyo, Japan) substrate by an RF magnetron sputtering system. Before the operating plasma, the substrates were cleaned with trichloroethylene, acetone, methanol, and distilled water (D.I. water), for 10 minutes respectively. The sputtering chamber was pumped down to $5 \times 10^{-6}$ torr by a turbo molecular pump. Highly pure (99.999\%) argon was applied as the sputtering gas, and the AZO (Al: 2 wt.\%) target had a diameter of 4 inches. Pre-sputtering treatment was performed to clean the target surface for 10 minutes by argon plasma. A distance of $6 \mathrm{~cm}$ was kept between the target and substrate. Furthermore, the substrate was rotated at $1,700 \mathrm{rpm}$. We changed the deposition RF power from $100 \mathrm{~W}$ to $350 \mathrm{~W}$ in steps of $50 \mathrm{~W}$, while the other conditions were fixed. The thickness of all films was kept constant at $150 \mathrm{~nm}$, so deposition time varied with the RF power.

The thickness and cross-sectional images of the films were checked by field emission scanning electron microscopy (FESEM; S-4800; Hitachi, Tokyo, Japan), and the crystallinity of the AZO film was analyzed with X-ray diffraction (XRD; Max $2500 H$; Rigaku, Tokyo, Japan). The electrical properties were investigated using a Hall measurement (HMS-3000; Ecopia, Arizona, USA) and a four-point probe (CMT-SR2000N; AIT, Suwon, Korea). The transmittance of the AZO films was investigated using an ultraviolet/visible (UV/VIS) spectrometer (S-3100; Scinco, Seoul, Korea).

\section{RESULTS AND DISCUSSION}

Figure 1 shows the growth rate of the AZO films as a function of RF power. The growth rate increased linearly as RF power increased. Based on this result, we fixed the film's

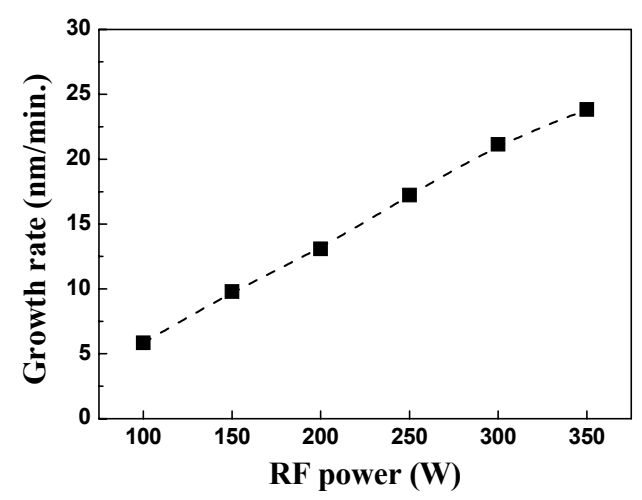

Fig. 1. The growth rate of Al-doped zinc oxide films as a function of radio frequency (RF) power.

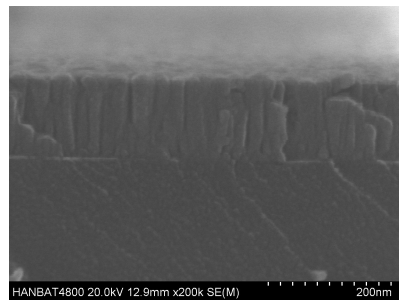

(a) $100 \mathrm{~W}$

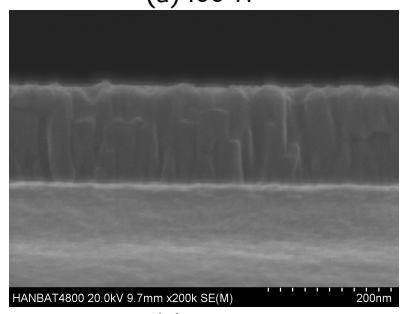

(c) $200 \mathrm{~W}$

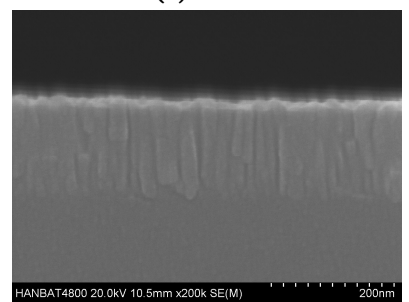

(e) $300 \mathrm{~W}$

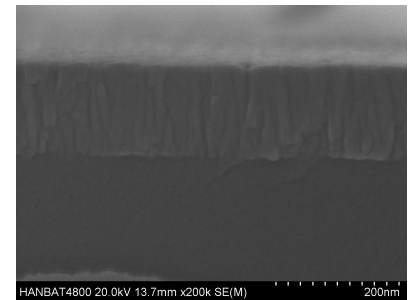

(b) $150 \mathrm{~W}$

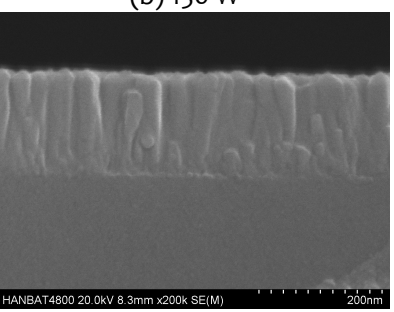

(d) $250 \mathrm{~W}$

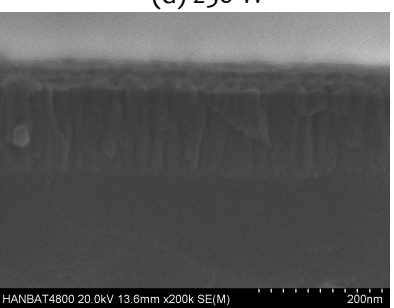

(f) $350 \mathrm{~W}$
Fig. 2. The cross-sectional scanning electron microscopy images of Al-doped zinc oxide films as a function of radio frequency power.

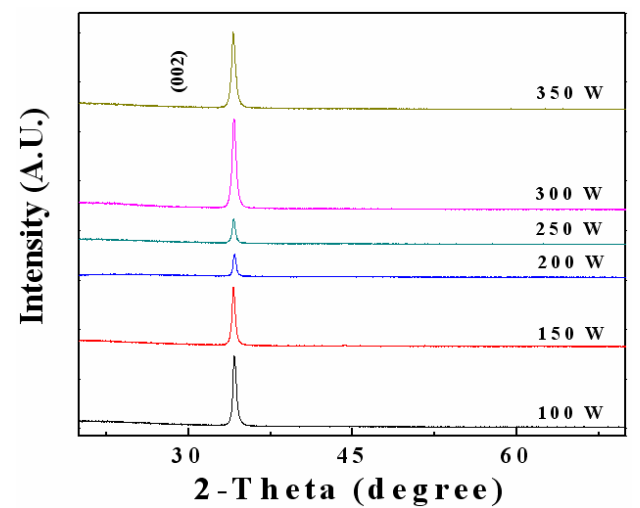

(a)

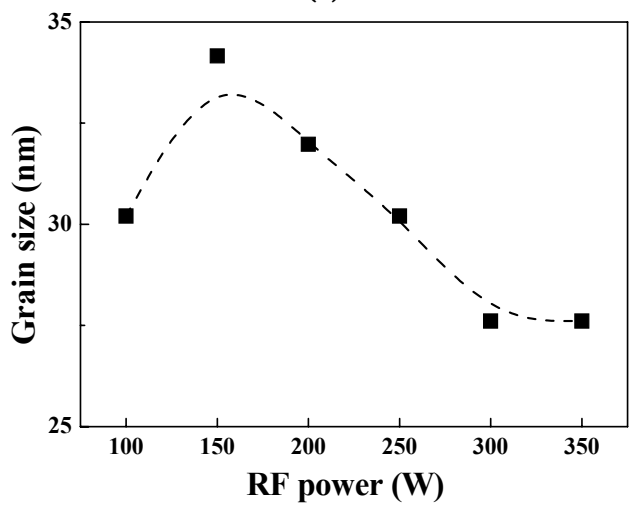

(b)

Fig. 3. X-ray diffraction results of Al-doped zinc oxide films deposited on a glass substrate, (a) X-ray spectra and (b) grain size. RF: radio frequency. 


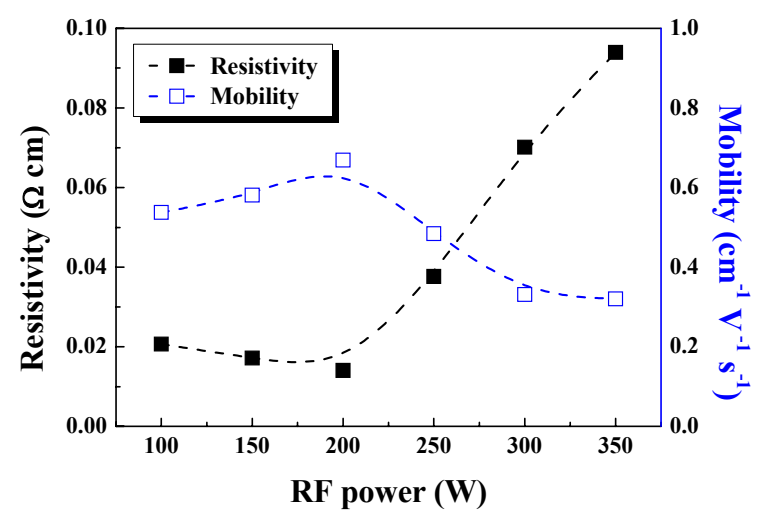

(a)

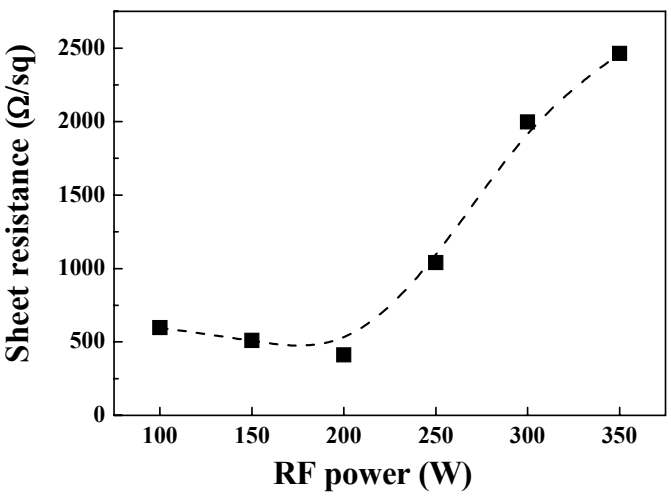

(b)

Fig. 4. Electrical properties of Al-doped zinc oxide films as a function of radio frequency (RF) power, (a) resistivity and mobility by Hall measurement and (b) sheet resistance by four-point probe.

thickness to $150 \mathrm{~nm}$ by adjusting the deposition time.

Figure 2 shows the cross-sectional SEM images of the AZO films as a function of RF power. The thicknesses of the films were $150 \mathrm{~nm}$. We confirmed that all films were grown as columnar structures, oriented around the c-axis.

Figure 3 shows the XRD patterns of the AZO films deposited on the glass substrate. Fig. 3(a) shows that all AZO films had highly preferred orientation (002) peaks. The intensities of the (002) direction of the main peaks decreased according to an increase of the RF powers from $100 \mathrm{~W}$ to $250 \mathrm{~W}$. However, in case of $300 \mathrm{~W}$ and $350 \mathrm{~W}$ of RF power, the intensities increased, and other (004) peaks were also observed. According to Scherrer's equation [14], the grain size could be calculated with the full width at half maximum (FWHM) value. The FWHM values of $100 \mathrm{~W}$ and $150 \mathrm{~W}$ were decreased from 0.31 to 0.26 , but the values between $200 \mathrm{~W}$ and $350 \mathrm{~W}$ increased from 0.28 to 0.35 . Fig. 3(b) shows the grain size of the AZO films as a function of the RF power calculated by Scherrer's equation. The grain sizes varied from $27.6 \mathrm{~nm}$ to $34.2 \mathrm{~nm}$ in the range of $100 \mathrm{~W}$ to $350 \mathrm{~W}$ RF power. We obtained the highest value of $34.2 \mathrm{~nm}$ at $150 \mathrm{~W}$ RF power.

Figure 4(a) shows the dependence of electrical resistivity and mobility as a function of RF power. The resistivity of the AZO films decreased when the RF power was increased from $100 \mathrm{~W}$ to $200 \mathrm{~W}$ and showed the lowest resistivity (0.014 $\Omega \cdot \mathrm{cm}$ ) at an RF power of $200 \mathrm{~W}$. However, the resistivity of the AZO films increased as the RF power increased from 250 $\mathrm{W}$ to $350 \mathrm{~W}$. On the contrary, Hall mobility increased from

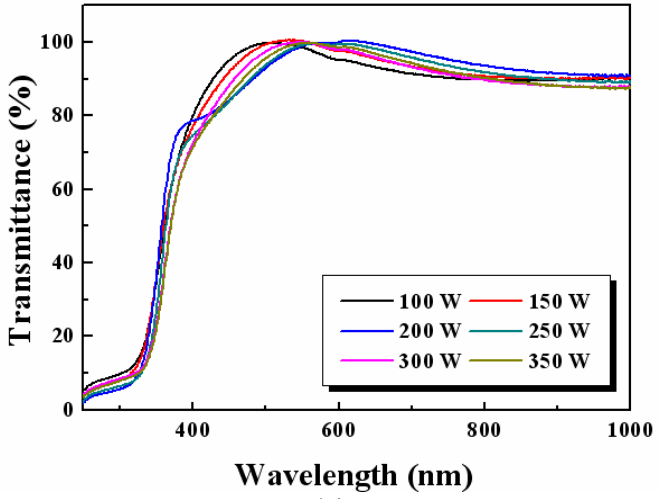

(a)

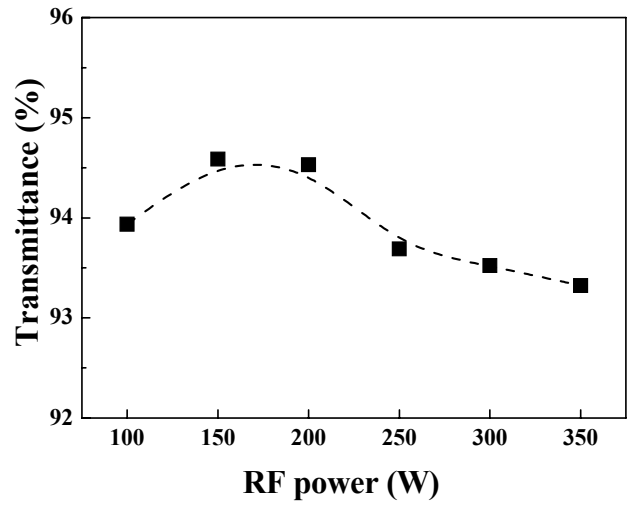

(b)

Fig. 5. Optical properties of Al-doped zinc oxide films as a function of radio frequency (RF) power, (a) transmittance spectra and (b) average transmittance (400-800 $\mathrm{nm})$.

$0.54 \mathrm{~cm}^{-1} \mathrm{~V}^{-1} \mathrm{~s}^{-1}$ to $0.67 \mathrm{~cm}^{-1} \mathrm{~V}^{-1} \mathrm{~s}^{-1}$ at the RF powers of $100 \mathrm{~W}$ to $200 \mathrm{~W}$. Fig. 4(b) shows the four-point probe results as a function of RF power. The sheet resistance of the AZO films decreased as the RF power increased from $100 \mathrm{~W}$ to $200 \mathrm{~W}$ and showed the lowest sheet resistance ( $411 \Omega \cdot s q)$ at an RF power of $200 \mathrm{~W}$. However, the resistivity of the AZO films increased as the RF power increased from $250 \mathrm{~W}$ to $350 \mathrm{~W}$. This result was entirely consistent with the Hall measurement results. As confirmed by XRD results, the crystallinity of the AZO films grown at room temperature was sensitive to the deposition RF power which indicated that the resistivity of the AZO films was influenced by the crystallinity.

Figure 5(a) shows transmittance spectra of AZO films measured by a UV-VIS spectrometer, and the average (400$800 \mathrm{~nm}$ ) transmittances are summarized in Fig. 5(b). The transmittance of the AZO films increased as the RF power increased from $100 \mathrm{~W}$ to $200 \mathrm{~W}$ and showed the highest resistivity (94.59\%) at the RF power of $150 \mathrm{~W}$. However, the transmittance of the AZO films decreased as the RF power increased from $250 \mathrm{~W}$ to $350 \mathrm{~W}$. All of the AZO films showed a high transmittance of over $90 \%$. The average transmittance was slightly changed by the RF power.

\section{CONCLUSIONS}

The AZO films were deposited on glass substrates by the RF magnetron sputtering method using an AZO target (Al: 2 
wt.\%) at room temperature. We investigated the effect of RF power on structural, electrical, and optical properties of 150nm-thick AZO films. XRD results showed that AZO films have highly preferred orientation (002) peaks. The grain size of the AZO film in the cases of $150 \mathrm{~W}$ and $200 \mathrm{~W}$ RF power were 27.6 and $34.2 \mathrm{~nm}$, respectively. Also, RF powers of $150 \mathrm{~W}$ and 200 $W$ provided good electrical and optical properties. The resulting values for $150 \mathrm{~W}$ and $200 \mathrm{~W}$ are summarized as a resistivity of $0.017 \Omega \cdot \mathrm{cm}$ and $0.014 \Omega \cdot \mathrm{cm}$, a sheet resistance of 510 $\Omega \cdot$ sq and $411 \Omega \cdot$ sq, and a transmittance $94.59 \%$ and $94.53 \%$, respectively.

\section{ACKNOWLEDGEMENTS}

This work was supported by the Korea Science and Engineering Foundation (KOSEF) grant funded by the Korea government (MEST) (No. 2009-0075246).

\section{REFERENCES}

[1] K. Matsubara, P. Fons, K. Iwata, A. Yamada, K. Sakurai, H. Tampo and S. Niki, Thin Solid Films 431-432, 369 (2003) [DOI: 10.1016/ s0040-6090(03)00243-8].

[2] C. P. Liu and G. R. Jeng, J. Alloys Compd. 468, 343 (2009) [DOI: 10.1016/j.jallcom.2008.01.053].
[3] K. Ellmer, J. Phys. D: Appl. Phys. 33, R17 (2000) [DOI: 10.1088/0022-3727/33/4/201].

[4] E. Fortunato, P. Barquinha, A. Pimentel, A. Gonçalves, A. Marques, L. Pereira, and R. Martins, Thin Solid Films 487, 205 (2005) [DOI: 10.1016/j.tsf.2005.01.066].

[5] H. Chen, H. J. Jin, C. B. Park, and G. C. Hoang, Trans. Electr. Electron. Mater. 10, 93 (2009).

[6] S. Zhao, Y. Zhou, Y. Liu, K. Zhao, S. Wang, W. Xiang, Z. Liu, P. Han, Z. Zhang, Z. Chen, H. Lu, K. Jin, B. Cheng, and G. Yang, Appl. Surf. Sci. 253, 726 (2006) [DOI: 10.1016/j.apsusc.2006.01. 010].

[7] B. T. Lee, T. H. Kim, and S. H. Jeong, J. Phys. D: Appl. Phys. 39, 957 (2006) [DOI: 10.1088/0022-3727/39/5/010].

[8] X. Feng, J. Phys.: Condens. Matter 16, 4251 (2004) [DOI: 10.1088/0953-8984/16/24/007].

[9] Y. Y. Liu, H. J. Jin, C. B. Park, and G. C. Hoang, Trans. Electr. Electron. Mater. 10, 24 (2009).

[10] J. Mass, P. Bhattacharya, and R. S. Katiyar, Mater. Sci. Eng., B 103, 9 (2003) [DOI: 10.1016/s0921-5107(03)00127-2].

[11] V. Musat, B. Teixeira, E. Fortunato, R. C. C. Monteiro, and P. Vilarinho, Surf. Coat. Technol. 180-181, 659 (2004) [DOI: 10.1016/ j.surfcoat.2003.10.112].

[12] A. N. Banerjee, C. K. Ghosh, K. K. Chattopadhyay, H. Minoura, A. K. Sarkar, A. Akiba, A. Kamiya, and T. Endo, Thin Solid Films 496, 112 (2006) [DOI: 10.1016/j.tsf. 2005.08.258].

[13] A. Geivandov, I. Kasianova, E. Kharatiyan, A. Lazarev, P. Lazarev, and S. Palto, EuroDisplay Digest (Moscow, Russia 2007 Aug. 1720, Crysoptix, KK.) p. 26.

[14] W. L. Bragg and W. H. Bragg, The Crystalline State (McMillan, New York, 1933) p. 189. 